\title{
- Potencial de utilização de resíduos da indústria papeleira como substratos paracultivo de Ganoderma lucidum (Reishi)
}

Débora Anzolin Valginhak ${ }^{1}$

Herta Stutz Dalla Santa²

\section{Resumo}

No Brasil, o Paraná é um dos estados que mais se destacam no setor de papel e celulose. A IBEMA Companhia Brasileira de Papel é a terceira maior fabricante de papel-cartão do país, responsável pela geração de lucros e empregos, mas também de resíduos, como os resíduos da área do pátio de madeira (VAR), a fibra da fábrica de pasta mecânica (FPM) e a fibra da estação de tratamento de efluentes (ETE). O cultivo de cogumelos em resíduos da indústria há muito tempo é avaliado, visto que esses organismos apresentam propriedades biodegradantes, alimentares e até medicinais. 0 objetivo deste trabalho foi caracterizar os resíduos da indústria papeleira e avaliar a aplicação destes e de suas misturas para a produção de cogumelos Ganoderma lucidum. A presença de elementos químicos foi determinada com uso de microscopia eletrônica de varredura acoplada a um detector de espectro de energia dispersiva. Após o preparo dos substratos em sacos plásticos, inoculação com grãos de trigo miceliados e incubação, foram avaliados o crescimento e vigor micelial e o surgimento de primórdios e basidiomas. Nenhum substrato apresentou elementos químicos prejudiciais ao ser humano. 0 resíduo FPM e o substrato padrão apresentaram crescimento micelial fortemente adensado, bem como a formação de primórdios e basidiomas aos 65 dias e 26 dias após a colonização dos substratos, respectivamente. O resíduo FPM serviu como substrato para o desenvolvimento de G. Iucidum, de forma que seu uso na produção do cogumelo possibilita agregar valor a este resíduo e reduzir sua disposição no meio ambiente.

Palavras-chave: Microbiologia ambiental. Papel e celulose. Cogumelo.

\section{Introdução}

O Brasil é um dos países que mais tem apresentado crescimento no setor de papel e celulose, sendo reconhecido como referência mundial devido às suas práticas sustentáveis (BIAZUS; HORA; LEITE, 2011), diferentemente de outros países que ainda fazem uso de florestas naturais, 100\% da produção de madeira é oriunda de florestas plantadas de pinus e eucalipto (SANTOS, F.; SANTOS, A.; SANTOS, M., 2013). Esse sucesso se deve principalmente à localização geográfica do país, que permite condições de clima, solo e luminosidade favoráveis, além dos investimentos na biotecnologia florestal (BIAZUS; HORA; LEITE, 2011).

Devido aos investimentos recebidos para modernização e ampliação do setor, o Brasil foi considerado em 2016 o segundo maior produtor de celulose do mundo e o oitavo maior produtor de

1 Universidade Estadual do Centro-Oeste. davalginhak@gmail.com. Rua Abgail Danguy Borges, 811, Bairro Conradinho, Guarapuava (PR), CEP: 85055-240

2 Universidade Estadual do Centro-Oeste, professora adjunta. hdalsanta@yahoo.com.br. 
papel, sendo que o setor madeireiro foi responsável por 0,9\% de toda a arrecadação de tributos do país (INDÚSTRIA BRASILEIRA DE ÁRVORES, 2017). Nesse cenário, o Paraná é um estado brasileiro que se destaca com uma área plantada de mais de 966 mil hectares, grande parte dessa área sendo ocupada por pinus, madeira de maior interesse no setor de celulose (ASSOCIAÇÃO PARANAENSE DE EMPRESAS DE BASE FLORESTAL, 2018).

A facilidade de se obter fontes de matéria-prima, a existência de recursos hidroelétricos próprios e a localização estratégica foram os motivos para a IBEMA Companhia Brasileira de Papel se instalar no Paraná (IBEMA, 2013). Fundada em 1956, a empresa localizada no município de Turvo é a terceira maior fabricante de papel-cartão do país, com produção anual de 140 mil toneladas de papel-cartão e responsável por 800 empregos (MEIRELLES, 2018).

De acordo com Kosztrzepa (2004), os processos produtivos industriais podem ser divididos em três fases: consumo de recursos, processamento e geração de produtos e subprodutos, e cada uma dessas fases causará danos ambientais. Nos processos industriais que envolvem madeira, como a produção de papel e celulose, são geradas grandes quantidades e variedades de resíduos com alto percentual de matéria orgânica (BELLOTE et al., 1998).

De acordo com a Indústria Brasileira de Árvores (2017), o setor madeireiro busca a gestão rigorosa dos seus resíduos sólidos, com redução da geração, destinação correta e atendimento aos requisitos legais. Porém, para que ocorra o descarte adequado de resíduos são necessárias grandes áreas para a construção de aterros, que geram despesas, provocando perdas econômicas e ambientais (PINTO, 2005).

Entre os resíduos gerados na empresa IBEMA Companhia Brasileira de papel, destacam-se os resíduos da área do pátio de corte da madeira (VAR), fibra da fábrica de pasta mecânica (FPM) e fibra da estação de tratamento de efluentes (ETE) (IBEMA, 2013). 0 resíduo de varredura é composto basicamente por casca de Pinus, acículas, areia e pedras presentes na área do pátio em que as madeiras são descascadas e cortadas até atingirem comprimentos menores, chamados cavacos. Os cavacos serão utilizados para a produção de uma pasta celulósica, que será empregada na fabricação de papel e durante o processo gerará o resíduo de fibra da fábrica de pasta mecânica (ASSOCIAÇÃO BRASILEIRA TÉCNICA DE CELULOSE E PAPEL, 1995). O resíduo da estação de tratamento de efluentes é obtido por meio da finalização de todos os processos de produção de papel, podendo conter resíduos de produtos químicos (IBEMA, 2013).

A crescente geração de resíduos nos diversos setores da indústria motivou a utilização destes resíduos para o cultivo de cogumelos. Esta alternativa de destinação dos resíduos mostrou-se eficiente e economicamente viável devido à facilidade do cultivo, ao baixo capital inicial investido e ao valor final dos cogumelos no mercado (PICCININ, 2000). Essa técnica também tem sido bastante estudada pelo fato de os cogumelos poderem atuar como biorremediadores, transformando os poluentes de diversos tipos de resíduos em produtos finais inócuos ou em constituintes do basidioma (SILVA; COELHO, 2006).

Os cogumelos são amplamente conhecidos pelas suas propriedades alimentares e medicinais, principalmente no Oriente (BERNARDI; MINOTTO; NASCIMENTO, 2008). Entre as espécies de cogumelos mais famosas atualmente, destacam-se o Ganoderma lucidum, conhecido há pelo menos 4000 anos pela medicina tradicional chinesa como o "rei" dos cogumelos medicinais (OLIVEIRA; CALADO; ROSADO, 2003; LÓPEZ, 2012). Por ser um fungo da podridão-branca, G. Iucidum possui alta capacidade de degradação da lignina e por este motivo seu crescimento na natureza ocorre em árvores de madeiras duras, como as coníferas (ROJAS, 2016). Atualmente, em cultivos comerciais, a forma mais utilizada para a produção de G. Iucidum é em serragem, com adição de farelo de trigo como fonte de nitrogênio, correção do $\mathrm{pH}$ a $5,5,95 \%$ de umidade e $28^{\circ} \mathrm{C}$ para formação de primór- 
dios (RAI, 2010). A colheita é realizada normalmente após três a seis meses de cultivo (CLEMENTINO; ROSADO, 2011).

O estudo de técnicas e utilização de diferentes tipos de resíduos no cultivo de cogumelos é muito importante, não apenas pela capacidade biorremediadora desses organismos, mas também devido ao crescente consumo de cogumelos pelos países ocidentais. Além disso, a ampla possibilidade de utilização de resíduos produzidos regionalmente levam à necessidade de expansão dos conhecimentos no que diz respeito a novas técnicas de cultivo de cogumelos, aliado ao intuito de solucionar o problema ambiental ocasionado pela disposição inadequada desses materiais no ambiente. Assim, diante do exposto, este trabalho teve como objetivo caracterizar diferentes resíduos da indústria papeleira, bem como avaliar o potencial de aplicação desses materiais como substratos para a produção de cogumelos G. Iucidum, possibilitando a agregação de valor a esses resíduos.

\section{Material e métodos}

Local de realização dos experimentos: todas as análises e experimentos foram realizados no Laboratório de Bioprocessos de Cogumelos e estufas correlatas do Departamento de Engenharia de Alimentos e no Laboratório Multiusuário de Microscopia Eletrônica do Departamento de Química da Universidade Estadual do Centro-Oeste.

Microrganismo e substratos utilizados: a cepa de cogumelo da espécie G. Iucidum foi cedida pela BRASMICEL (Suzano-SP) e os resíduos utilizados como substratos para o cultivo do cogumelo foram cedidos pela IBEMA Companhia Brasileira de Papel. Como substrato padrão foi utilizada serragem de Pinus sp. (SER) adquirida em madeireira da região.

Produção de sementes: para a produção de "sementes de cogumelo" foram utilizados grãos de trigo, os quais foram imersos em água durante 12 horas, escorridos e distribuídos em pacotes plásticos vedados com espuma, sendo esterilizados por 30 minutos, a $121{ }^{\circ} \mathrm{C}$. Os pacotes foram inoculados com suspensão de micélio e incubados a $25^{\circ} \mathrm{C}$ até completa colonização micelial do substrato (OEI, 2006).

\section{Análises físico-químicas:}

Determinação de elementos químicos: os elementos químicos presentes nos resíduos foram determinados qualitativa e semiquantitativamente por meio de microscópio eletrônico de varredura (MEV), acoplado a um detector de espectro de energia dispersiva (EDS). Essa análise foi realizada a partir de uma visão ampla em todos os resíduos e em determinados pontos, quando se verificavam regiões morfologicamente diferentes do habitual.

Determinação do pH: em um copo béquer de $250 \mathrm{~mL}, 10 \mathrm{~g}$ de amostra dos resíduos e 100 $\mathrm{mL}$ de água anteriormente fervida e resfriada foram adicionados. 0 conteúdo foi deixado no frasco por 30 minutos, sendo agitado a cada 5 minutos. Posteriormente, o frasco foi deixado em repouso por 10 minutos e, após, o conteúdo foi filtrado para outro béquer, sendo realizada a medição de $\mathrm{pH}$ no líquido restante com o auxílio de um pHmêtro digital. A análise foi realizada em triplicata (INSTITUTO ADOLFO LUTZ, 2008). 
Preparo dos substratos, inoculação e incubação: os substratos e suas misturas foram umedecidos por 4 horas em água com adição de 0,9\% de ureia, escorridos e suplementados com 10\% de farelo de trigo. Em seguida, os substratos foram embalados até ocupar 2/3 do volume útil de pacotes de polipropileno, que foram fechados com algodão e esterilizados por 40 minutos, a $121{ }^{\circ} \mathrm{C}$ (BELLETTINI, 2014).

O experimento foi realizado com 11 tratamentos (Tabela 1) em triplicata.

Tabela 1. Resíduos da indústria papeleira e misturas de resíduos utilizados como substratos para cultivo de Ganoderma lucidum.

\begin{tabular}{ccccc}
\hline \multirow{2}{*}{ Tratamentos } & \multicolumn{5}{c}{ Resíduos (\%) } \\
\cline { 2 - 5 } & SER $^{*}$ & VAR $^{*}$ & FPM $^{*}$ & ETE* $^{*}$ \\
\hline 1 & 100 & - & - & - \\
3 & - & 100 & - & - \\
4 & - & - & 100 & 100 \\
5 & - & - & - & - \\
6 & 50 & 50 & - & - \\
7 & 50 & - & 50 & 50 \\
8 & 50 & - & - & - \\
9 & - & 50 & 50 & 50 \\
10 & - & 50 & - & 50 \\
11 & - & - & 50 & 25 \\
\hline
\end{tabular}

* SER- Serragem; VAR- Resíduo de Varredura da Área do Pátio da Madeira; FPM- Fibra da Fábrica de Pasta Mecânica; ETE- Fibra da Estação de Tratamento de Efluentes.

Fonte: Elaboração das autoras (2014)

Os tratamentos foram: (1) 100\% SER; (2) 100\% VAR; (3) 100\% FPM; (4) 100\% ETE; (5) $50 \%$ SER e 50\% VAR; (6) 50\% SER e 50\% FPM; (7) 50\% SER e 50\% ETE ; (8) 50\% VAR e 50\% FPM; (9) 50\% VAR e 50\% ETE; (10) 50\% FPM e 50\% ETE; (11) 25\% SER, VAR, FPM e ETE.

Os pacotes preparados para cada tratamento foram inoculados com $10 \% \mathrm{v} / \mathrm{v}$ de semente e incubados em temperatura de $25( \pm 2)^{\circ} \mathrm{C}$ em ambiente sem luminosidade.

\section{Análises de crescimento do fungo}

Velocidade de crescimento: medições diárias com paquímetro metálico foram realizadas até a completa colonização do substrato ou até a verificação da estagnação do crescimento fúngico.

Vigor micelial: foi avaliado pelo critério subjetivo de notas em que: nota $1=$ fracamente adensado; nota 2 = mediamente adensado e nota 3 = fortemente adensado (PEDRA; MARINO, 2006). 
Surgimento de primórdios e frutificação: o tempo para surgimento dos primórdios foi contabilizado desde a completa colonização micelial até a emissão dos primórdios e formação de basidiomas.

\section{Resultados e discussão}

\section{Análises físico-químicas}

Determinação de elementos químicos

Na Figura 1 estão as imagens dos diferentes resíduos obtidas pelo MEV com visão geral para todos e visão pontual quando se observaram componentes morfologicamente diferentes do obtido na visão geral.

Figura 1. Imagens obtidas pelo microscópio eletrônico de varredura (aumento de 400x) da serragem de pinus (SER), resíduo da área do pátio de corte da madeira (VAR), fibra da fábrica de pasta mecânica (FPM) e fibra da estação de tratamento de efluentes (ETE).
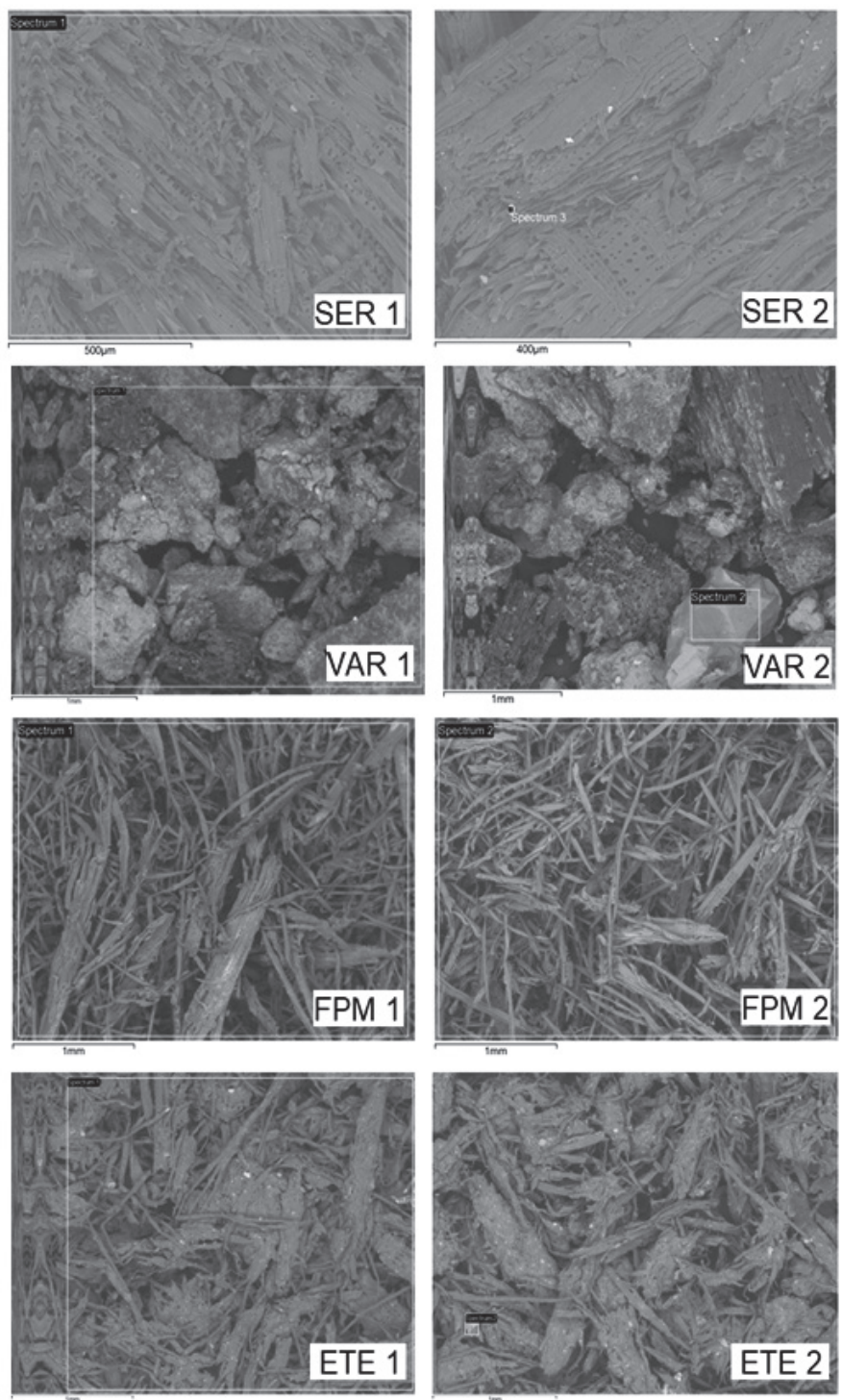

* 1 Visão geral, 2 visão pontual

Fonte: Elaboração das autoras (2014) 
Na Tabela 2, encontram-se os resultados da análise de elementos químicos obtidos pelo EDS.

Tabela 2. Composição química (em \%) dos resíduos da indústria papeleira obtidos pelo EDS.

\begin{tabular}{|c|c|c|c|c|c|c|c|c|}
\hline \multirow{3}{*}{ Elementos } & \multicolumn{8}{|c|}{ Resíduos (em \%) } \\
\hline & \multicolumn{2}{|c|}{$\begin{array}{l}\text { SER } \\
\end{array}$} & \multicolumn{2}{|c|}{ VAR } & \multicolumn{2}{|c|}{ FPM } & \multicolumn{2}{|c|}{ ETE } \\
\hline & $1 *$ & $2 *$ & $1 *$ & $2 *$ & $1 *$ & $2 *$ & $1 *$ & $2 *$ \\
\hline Carbono & 54,07 & 32,58 & 29,22 & 25,63 & 51,50 & 51,77 & 48,86 & 42,71 \\
\hline Oxigênio & 44,06 & 43,28 & 47,37 & 52,84 & 48,50 & 48,23 & 48,2 & 48,02 \\
\hline Silício & - & 10,81 & 5,52 & 14,17 & - & - & 0,79 & 4,28 \\
\hline Alumínio & 1,86 & 8,94 & 5,45 & 2,93 & - & - & 0,64 & 4,22 \\
\hline Magnésio & - & 0,58 & - & - & - & - & - & 0,21 \\
\hline Ferro & - & 1,25 & - & 4,43 & - & - & - & - \\
\hline Titânio & - & - & 11,58 & - & - & - & - & - \\
\hline Potássio & - & 2,56 & - & - & - & - & - & - \\
\hline Cálcio & - & - & - & - & - & - & 1,16 & 0,55 \\
\hline Enxofre & - & & 0,87 & - & - & - & - & - \\
\hline
\end{tabular}

*1 Análise de visão geral, 2 Análise de visão pontual, com aumento de 400 X.

Fonte: Elaboração das autoras (2014)

Os componentes encontrados em maior quantidade foram o carbono e o oxigênio, resultado já esperado pelo fato de os resíduos serem provenientes de compostos orgânicos.

O resíduo FPM apresentou apenas esses dois elementos em sua constituição. Esse resíduo é formado basicamente de lignina, macromolécula que apresenta apenas carbono, oxigênio e hidrogênio em sua composição química e é gerado por meio do processo Stone Ground-Wood (SGW), que realiza o desfibramento mecânico das toras de madeira para obtenção da pasta celulósica (CAMPOS, 2010), que será utilizada na fabricação do papel-cartão.

O silício foi o elemento encontrado em terceira maior quantidade nos resíduos VAR, SER e ETE. Esse elemento é encontrado em proporções consideráveis na composição de diversos tipos de rochas, areia e solos (PEIXOTO, 2001), podendo estar associado às madeiras que chegam na indústria. Segundo a Associação Brasileira Técnica de Celulose e Papel (1995), a pasta celulósica sofre inúmeras lavagens para retiradas de contaminantes, como areia, sendo que o líquido resultante dessas lavagens é destinado para a estação de tratamento de efluentes.

O alumínio também foi observado nos resíduos de SER, VAR e ETE. Esse elemento foi encontrado em grandes proporções em determinados pontos das amostras de SER quando comparado com a visão ampla, sugerindo que esse mineral tenha sido acumulado como resíduo do maquinário utilizado durante o corte da madeira. Sua presença no resíduo menos processado VAR pode representar a associação com solos, pois, entre os elementos metálicos, o alumínio é o mais abundante na crosta terrestre (COMPANHIA AMBIENTAL DO ESTADO DE SÃO PAULO, 2014).

O enxofre, magnésio e cálcio são elementos muito importantes na nutrição de plantas, considerados como macronutrientes secundários e presentes em fertilizantes (UNIVERSAL DE FERTILIZANTES S.A., 2012). Por este motivo, a presença desses compostos nos resíduos menos processados pode sugerir sua presença na composição das plantas. 0 magnésio e o potássio, na forma de sulfato 
de magnésio e fosfato de potássio, têm sido empregados na formulação de meio líquido para cultivo submerso de fungos basidiomicetos (FAN, 2002), na concentração de 0,3 g L-1 e 0,5 g L-1, respectivamente. Da mesma forma, estes podem ter favorecido o crescimento micelial de G. Iucidum nos substratos SER e ETE.

No resíduo sem processamento VAR, foi encontrada uma baixa porcentagem de titânio, nono elemento mais abundante da terra e não tóxico (MAIA, 2001). Também verificou-se a presença de pequena quantidade de ferro nas amostras de SER e VAR, metal que possui uma grande importância no organismo humano, tanto na sua condição atômica quanto associado a outras substâncias (SANTOS; DONADIA; SANTOS, 2010).

Apesar de ser o elemento mais abundante na natureza, a porcentagem do elemento hidrogênio não pode ser determinada por essa metodologia devido ao seu baixo peso molecular (DEDAVID; GOMES; MACHADO, 2007).

\section{Determinação do pH}

A determinação do valor de $\mathrm{pH}$ indicou o caráter ácido do substrato SER $(5,7)$ e básico do FPM $(7,9)$. Os resíduos de ETE e VAR apresentaram pH de 7,5 e 6,3, respectivamente. Hsieh, Hsu e Yang (2005) analisaram que a faixa de $\mathrm{pH}$ para crescimento de G. Iucidum em vinhaça foi de 4 a 7, apresentando maior crescimento em $\mathrm{pH} 5$.

\section{Análises de crescimento do fungo}

\section{Velocidade de crescimento}

Os dados da velocidade de crescimento micelial foram divididos em duas figuras para melhor entendimento.

Os melhores valores foram observados no tratamento 1 e 4 (Figura 2). Quando apenas SER foi empregada como substrato, a completa colonização micelial do saco plástico foi alcançada após 48 dias de crescimento, correspondendo a $170 \mathrm{~mm}$. Quando apenas ETE foi utilizada, a completa colonização micelial foi observada após 65 dias, apresentando um crescimento de $182 \mathrm{~mm}$. 
Figura 2. Velocidade do crescimento micelial (mm/dia) de G. Iucidum nos tratamentos 1, 2, 3 e 4.

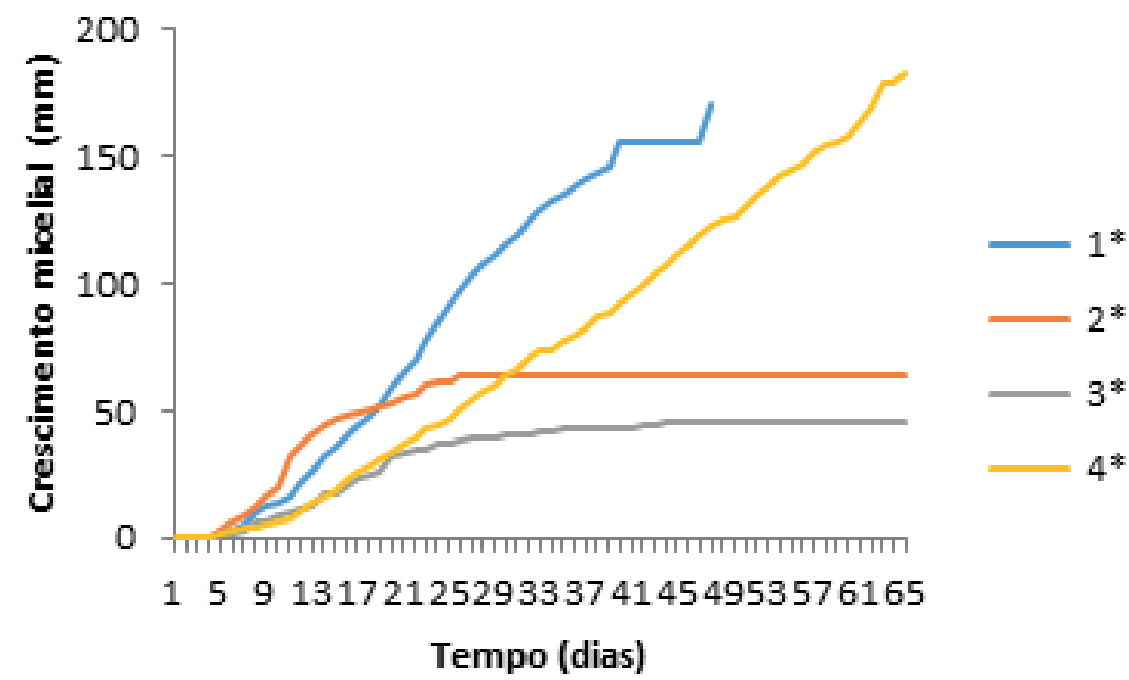

* Tratamentos: (1) 100\% SER; (2) 100\% VAR; (3) 100\% FPM; (4) 100\% ETE.

Fonte: Elaboração das autoras (2014)

Houve estagnação do crescimento micelial, como apresentado na Figura 3, para todos os tratamentos contendo a mistura entre os resíduos.

Figura 3. Velocidade do crescimento micelial (mm/dia) de G. Iucidum nos tratamentos 5, 6, 7, 8, 9, 10 e 11.

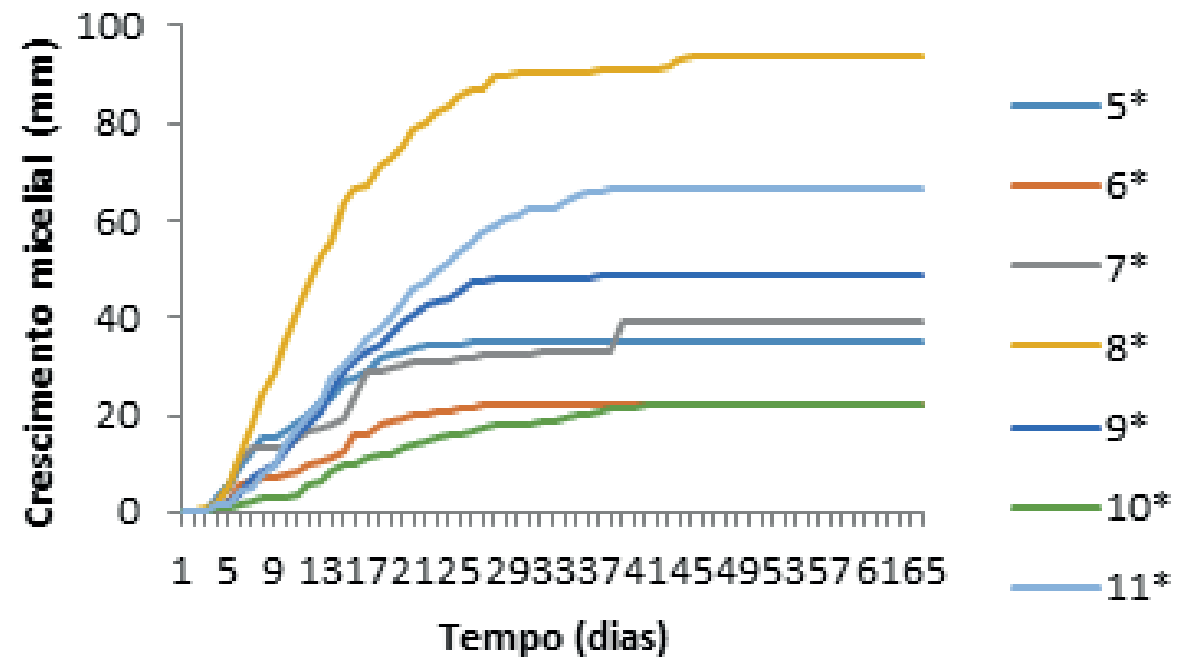

* Tratamentos: (5) 50\% SER e 50\% VAR; (6) 50\% SER e 50\% FPM; (7) 50\% SER e $50 \%$ ETE ; (8) $50 \%$ VAR e $50 \%$ FPM; (9) 50\% VAR e 50\% ETE; (10) 50\% FPM e 50\% ETE; (11) 25\% SER, VAR, FPM e ETE.

Fonte: Elaboração das autoras (2014)

Segundo Gomes-da-Costa, Coimbra e Silva (2008), Sales-Campos e Andrade (2011), o tipo de substrato e de suplementação pode influenciar a velocidade do crescimento micelial. Apesar de a suplementação não ter sido avaliada no presente estudo, bons índices de crescimento de G. Iucidum 
foram observados com a utilização apenas da SER e da ETE (tratamentos 1 e 4). Diversos estudos na literatura demonstraram que a suplementação pode significativamente elevar a taxa de crescimento apresentada por basidiomicetos, incluindo G. Iucidum. Macedo et al. (2011) verificaram que este fungo obteve melhor crescimento em casca de cupuaçu e serragem, suplementadas com $10 \%$ e 20\% de farelo de arroz, devido ao aumento na disponibilidade de nutrientes facilmente assimiláveis. Alananbeh, Bougellah e Kaff (2014) verificaram que o crescimento micelial de Pleorotus ostreatus foi favorecido quando palhas de trigo suplementaram o substrato de cultivo constituído inicialmente apenas por folhas de palmeira.

Apesar de G. Iucidum ser um fungo capaz de liberar enzimas capazes de converter substratos ligninocelulósicos em moléculas menores, vários são os fatores que estão associados a essa capacidade. Li, Zhou e Li (2007) observaram que o crescimento de linhagens de Ganoderma foi mais significativo na presença de sais minerais como sulfato de magnésio, fosfato de potássio e cloreto de cálcio, sugerindo que a falta de nutrientes pode ter provocado resultados baixos de crescimento no resíduo FPM.

Em alguns tratamentos ocorreu estagnação do crescimento micelial e o fungo não colonizou nem metade do substrato, conforme pode ser visualizado na Figura 4, que mostra o tratamento 9 com crescimento estagnado aos 147 dias e no tratamento 4 com crescimento micelial completo após apenas 65 dias.

Figura 4. Crescimento micelial de G. Iucidum: (A) tratamento 9 após 147 dias de incubação; (B) tratamento 4 após 65 dias de incubação.

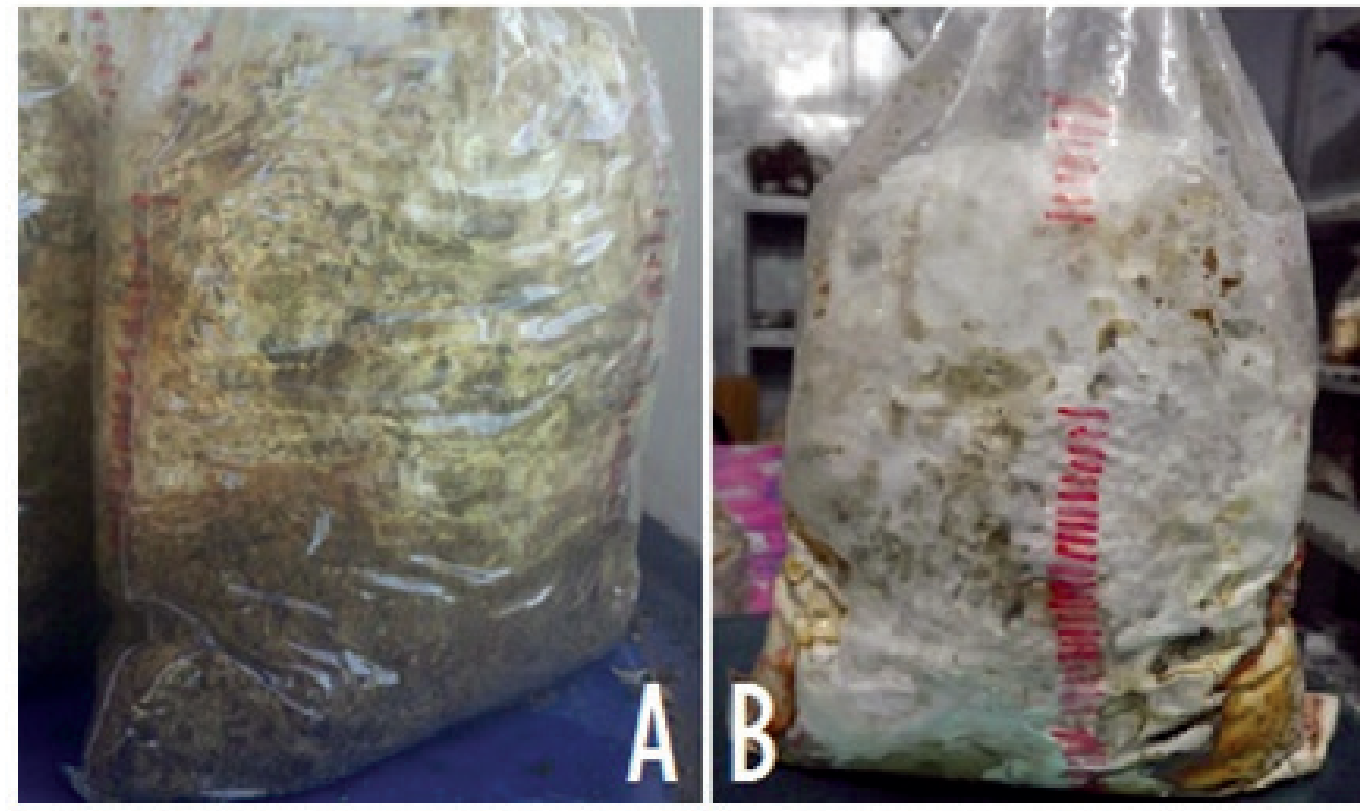

Fonte: Elaboração das autoras (2014)

\section{Vigor micelial}

O vigor micelial de G. Iucidum só foi considerado fortemente adensado nos tratamentos 1 e 4 (Tabela 3). Esse resultado é relevante, pois o vigor do micélio está diretamente relacionado com a capacidade de crescimento e utilização dos nutrientes do meio de cultivo (MACEDO et al., 2011). 
Maziero, Bononi e Capelari (1990) e Ragunathan et al. (1996) afirmaram que o vigor também é um dos parâmetros que podem ser influenciados pela composição do substrato, pelos isolados testados e pela suplementação fornecida. Ao avaliarem o cultivo de Pleurotus spp. em serragem de casca de coco, Pedra e Marino (2006) constataram que os tratamentos que apresentaram maior vigor micelial também foram os mais produtivos e os mais precoces na indução de primórdios.

Tabela 3. Vigor micelial de G. Iucidum em resíduos da indústria papeleira

\begin{tabular}{ccc}
\hline Vigor micelial & Nota & Tratamentos \\
\hline Fracamente adensado & 1 & $2,3,5,7,10$ \\
Mediamente adensado & 2 & $6,8,9,11$ \\
Fortemente adensado & 3 & 1,4 \\
\hline
\end{tabular}

Fonte: Elaboração das autoras (2014)

\section{Surgimento de primórdios e frutificação}

Em relação ao surgimento dos primórdios de G. Iucidum, verificou-se que apenas os tratamentos 1 e 4 tiveram resultados positivos (Figura 5). No tratamento 1, o processo ocorreu 26 dias após a colonização do substrato com três frutificações, enquanto que no tratamento 4 o surgimento de primórdios ocorreu 65 dias após a colonização, apresentando uma frutificação. Alguns trabalhos relatam um número maior de frutificações, o que pode indicar maior produtividade (COLAUTO; EIRA; MINHONI, 1998).

Figura 5. Primórdios e formação de basidiomas de G. Iucidum em resíduos da indústria papeleira. (A) Tratamento 1; (B) Tratamento 4.

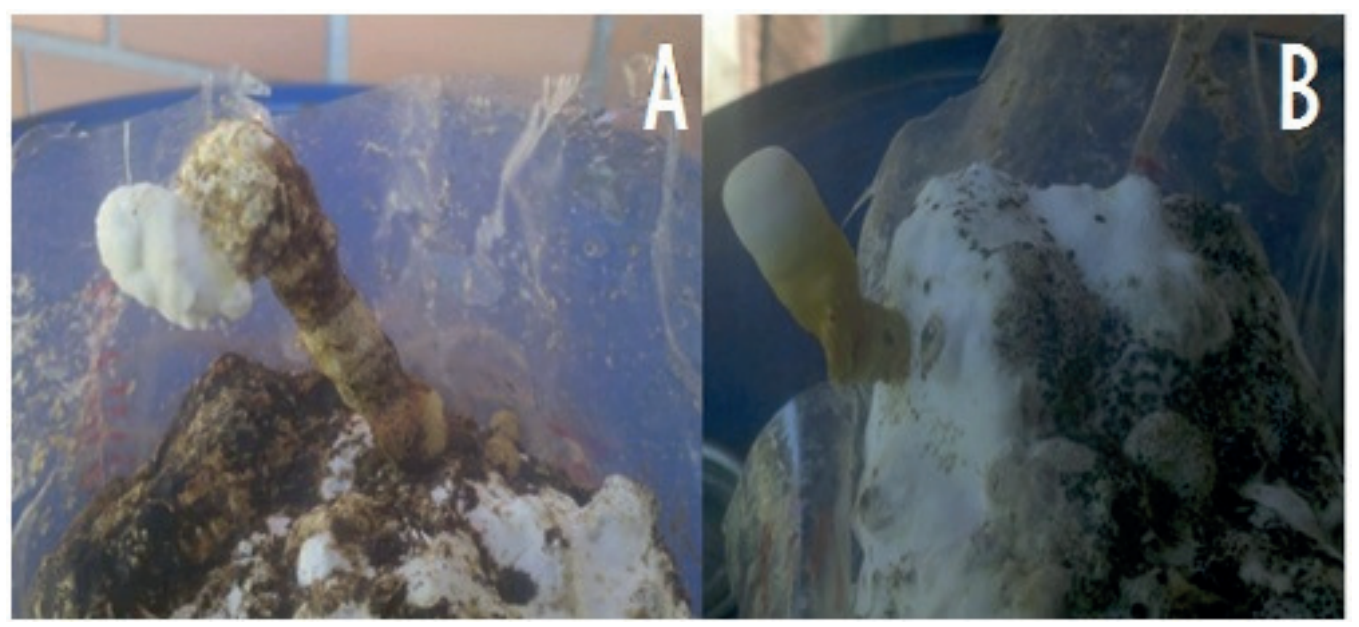

Fonte: Elaboração das autoras (2014)

$\mathrm{O} \mathrm{pH}$ da SER, por ser levemente ácido, é considerado ideal para o crescimento fúngico, fator que deve ter contribuído para o bom desenvolvimento de G. Iucidum na serragem. Apesar de a ETE não apresentar $\mathrm{pH}$ ideal para o desenvolvimento fúngico, um bom crescimento micelial foi verificado com a utilização deste substrato, porém em maior tempo de crescimento quando comparado ao crescimento observado em presença de SER. Neste último caso, apesar de o substrato apresentar um pH mais neutro $(7,5)$, G. Iucidum apresentou um bom crescimento, sendo este superior ao observado 
em presença de VAR, o qual apresentou um valor de $\mathrm{pH}$ mais ácido $(6,3)$. Por esta razão, sugere-se que outros fatores como a constituição nutricional desses materiais tenham influenciado as taxas de crescimento de G. lucidum.

\title{
Conclusão
}

O uso de resíduos da indústria papeleira como substratos para o cultivo do cogumelo G. Iucidum resultou em bom desenvolvimento nos tratamentos 1 e 4, constituídos de 100\% SER e 100\% ETE, respectivamente. Os tratamentos 2, 3, 5, 6, 7, 8, 9, 10 e 11 apresentaram estagnação do crescimento e não foram capazes de induzir a formação de basidiomas, sendo considerados inviáveis para tal utilização.

Mesmo não apresentando todas as características ideais ao crescimento fúngico, a utilização de $100 \%$ do resíduo ETE obteve as melhores taxas de crescimento, vigor micelial e formação de primórdios. Esse resultado mostra que vários são os fatores que, unidos, estão relacionados com a capacidade de cultivo de cogumelos de determinados substratos.

Assim, conclui-se que o ETE, entre os resíduos testados, é promissor para ser empregado como substrato para o cultivo de G. Iucidum. Entretanto, esse resíduo e os basidiomas gerados deverão passar por outras análises para determinar a segurança da utilização deste resíduo no referido processo.

\section{Potential use of paper industry waste as substrates for cultivation of Ganoderma lucidum (Reishi)}

\begin{abstract}
In Brazil, Paraná is one of the states that receive the most attention in the pulp and paper sector. IBEMA Companhia Brasileira de Papel is the third largest paperboard manufacturer in the country, responsible for generating profits and jobs; however, it also generates waste, such as the waste of the wooden patio area (VAR), the pulp mill fiber (FPM) and fiber from the effluent treatment plant (ETE). The cultivation of mushrooms in industrial waste has long been evaluated, since these organisms have biodegrading, food and even medicinal properties. The objective of this work was to characterize the residues of the paper industry and to evaluate their application and mixtures for the production of Ganoderma lucidum mushrooms. The presence of chemical elements was determined by the use of a scanning electron microscopy coupled to a dispersive energy spectrum detector. After the preparation of the substrates in plastic bags, inoculation with micellar wheat grains and incubation, mycelial growth and vigor, the emergence of primordia and basidiomas were evaluated. No substrate presented chemical elements harmful to the human being. The FPM residue and the standard substrate had strongly mixed mycelial growth, as well as the formation of primordia and basidiomas at 65 days and 26 days after colonization of the substrates, respectively. The FPM residue served as a substrate for the development of G. Iucidum, so that its use in the production of the mushroom would allow to add value to this residue and reduce its disposition in the environment.
\end{abstract}

Keywords: Environmental microbiology. Pulp and paper. Mushrooms. 


\section{Referências}

ALANANBEH, K. M.; BOUGELLAH, N. A.; KAFF, N. S. Cultivation of oyster mushroom Pleorotus ostreatus on date-palm leaves mixed with other agro-wastes in Saudi Arabia. Said Journal of Biological Sciences, Riyadh, v. 21, p. 616-625, 2014. Disponível em: < https://www. sciencedirect.com/science/article/pii/S1319562X1400093X>. Acesso em: 7 set. 2018.

ASSOCIAÇÃO BRASILEIRA TÉCNICA DE CELULOSE E PAPEL. Curso de pasta mecânica. Monte Alegre: ABTCP, 1995. p. 16-21. Disponível em: <http://www.celso-foelkel.com.br/ artigos/outros/07_Curso\%20Pasta\%20Mecanica.pdf>. Acesso em: 7 set. 2018.

ASSOCIAÇÃO PARANAENSE DE EMPRESAS DE BASE FLORESTAL. Estudo setorial. Curitiba: APRE, 2018. p. 28-30. Disponível em: <http://www.apreflorestas.com.br/wp-content/ uploads/2018/02/Estudo-Setorial-2018_APRE.pdf>. Acesso em: 8 set. 2018.

BELLETTINI, M. B. Desenvolvimento de um bioprocesso integrado para valorização de bainha de pupunha (Bactris gasipaes Kunth): Produção de cogumelos (Pleurotus spp.) e alface (Lactuta sativa) cv. Verônica. 2014. 134 f. Dissertação (Mestrado em Engenharia de Alimentos)- Universidade Federal do Paraná, Curitiba, 2014.

BELLOTE, A. F. J.; SILVA, H. D.; FERREIRA, C. A.; ANDRADE, G. C. Resíduos da indústria de celulose em plantios florestais. Boletim de Pesquisa Florestal, Colombo, n. 37, p. 99-106, 1998. Disponível em: <https://www.embrapa.br/busca-de-publicacoes/-/publicacao/282198/ residuos-da-industria-de-celulose-em-plantios-florestais > . Acesso em: 7 set. 2018.

BERNARDI, E.; MINOTTO, E.; NASCIMENTO, J. S. Aproveitamento do resíduo de curtume como suplemento no cultivo de Pleurotus ostreatus. Arquivos do Instituto de Biologia, São Paulo, v. 75, n. 2, p. 243-246, 2008. Disponível em: <http://www.biologico.sp.gov.br/ uploads/docs/arq/v75_2/bernardi.pdf>. Acesso em: 7 set. 2018.

BIAZUS, A.; HORA, A. B.; LEITE, B. G. P. O potencial de investimento nos setores florestal, de celulose e de papel. In: TORRES, E.; PUGA, F.; MEIRELLES, B. Perspectivas do investimento 2010-2013. Rio de Janeiro: BNDES, 2011. p. 108-143.

CAMPOS, E. S. Curso básico de fabricação de papel com ênfase nas propriedades dos papéis de fibra curta. Aracruz: ABTCP, 2010. p. 26. Disponível em: < http://www.eucalyptus.com. br/artigos/outros/2010_Papel_Fibra_Curta.pdf>. Acesso em: 7 set. 2018.

CLEMENTINO, L. S.; ROSADO, F. R. Cultivo submerso de Ganoderma lucidum (Curt. ex Fr.) Karst em diferentes meios de cultura. Revista em Agronegócios e Meio Ambiente, Maringá, v. 4, n. 1, p. 131-144, 2011. Disponível em: <http://periodicos.unicesumar.edu.br/index.php/rama/article/ view/1253/1190>. Acesso em: 7 set. 2018.

COLAUTO, N. B.; EIRA A. F.; MINHONI, M. T. A. Fatores físicos que afetam a produtividade do cogumelo comestível Pleurotus sajor - caju (Fr.) Singer. Cientifica, v. 26, n. 1-2, p. 25-43, 1998. Disponível em: $\quad<$ https://www.researchgate.net/publication/262687398_Fatores_fisicos_que_afetam_a_ produtividade_do_cogumelo_comestivel_Pleurotus_sajor-caju_Fr_Singer>. Acesso em: 7 set. 2018. 
COMPANHIA AMBIENTAL DO ESTADO DE SÃO PAULO. Ficha de informação toxicológica: alumínio. São Paulo: CETESB, 2014. Disponível em: https://www.cetesb.sp.gov.br/laboratorios/wp-content/ uploads/sites/24/2013/11/Aluminio.pdf. Acesso em: 7 set. 2018.

DEDAVID, B. A.; GOMES, C. I.; MACHADO, G. Microscopia eletrônica de varredura: aplicações e preparação de amostras: materiais poliméricos, metálicos e semicondutores. São Paulo: EDIPUCRS, 2007. p. 22.

FAN, L. Production of extra-cellular polysaccharide from Agaricus blazei by submerged and solid state culture and its antitumor effect. 2002. $111 \mathrm{f}$. Tese (Doutorado em Processos Biotecnológicos) - Universidade Federal do Paraná, Curitiba, 2002.

GOMES-DA-COSTA, S. M.; COIMBRA, L. B.; SILVA, E. S. Crescimento micelial de dois isolados de Lentinula edodes (Berk.). Pegler em resíduos lignocelulóticos. Acta Scientiarum. Biological Sciences, Maringá, v. 30, n. 2, p. 192-196, 2008. Disponível em: <http://periodicos.uem.br/ojs/index.php/ ActaSciBiolSci/article/view/3622>. Acesso em: 7 set. 2018.

HSIEH, C.; HSU, T.; YANG, F. Production of polysaccharides of Ganoderma lucidum (CCRC36021) by reusing thin stillage. Process Biochemistry, Hamburg, v. 40, n. 2, p. 909-916, 2005. Disponível em: <https://www.sciencedirect.com/science/article/pii/S0032959204000998>. Acesso em: 7 set. 2018.

IBEMA - COMPANHIA BRASILEIRA DE PAPEL. Histórico e dados gerais da empresa [mensagem pessoal]. Mensagem recebida por <italo.tadeu2010@hotmail.com> em 28 ago. 2013.

INDÚSTRIA BRASILEIRA DE ÁRVORES. Relatório 2017. São Paulo: Ibá, 2017. p. 41-65. Disponível em: <http://iba.org/images/shared/Biblioteca/IBA_RelatorioAnual2017.pdf>. Acesso em: 8 set. 2018.

INSTITUTO ADOLFO LUTZ. Métodos químicos e físicos para análise dos alimentos. São Paulo: Instituto Adolfo Lutz, 2008. p. 104.

KOSZTRZEPA, R. O. Evidenciação dos eventos relacionados com o meio ambiente: um estudo em indústrias químicas. 2004. 156 f. Dissertação (Mestrado em Ciências Contábeis) - Universidade do Rio dos Sinos, São Leopoldo, 2004.

LI, X. L.; ZHOU, A. G.; LI, X. M. A. Inhibition of Lycium barbarum polysaccharides and Ganoderma lucidum polysaccharides against oxidative injury induced by $\gamma$-irradiation in rat liver mitochondria. Carbohydrate Polymers, Worcester, v. 69, n. 1, p. 172-178, 2007. Disponível em: <https://www. sciencedirect.com/science/article/pii/S014486170600467X>. Acesso em: 7 set. 2018.

LÓPEZ, D. R. D. Obtención de cepas silvestres de Ganoderma lucidum y la caracterización de una para la cuantificación de exopolisacáridos en cultivo de células em suspension. 2012. 60 f. Tesis (Licenciatura em Biología) - Universidad de Guadalajara, Jalisco, 2012.

MACEDO, A. J. P.; KIRSCH, L. S.; PALHETA, R. A.; PUTZKE, J.; TEIXEIRA, M. F. S. Crescimento micelial de Ganoderma lucidum (Curt.: Fr.) P. Karst. em resíduos lignocelulósicos disponíveis na Amazônia. Caderno de Pesquisa, Série Biologia, Santa Cruz do Sul, v. 23, n. 3, p. 16-25, 2011. Disponível em: <https://online.unisc.br/seer/index.php/cadpesquisa/article/view/4384>. Acesso em: 7 set. 2018. 
MAIA, A. Titânio. In: TUNES, M. R. Balanço Mineral Brasileiro. Brasília: DNPM, 2001. Disponível em: $\quad<$ http://www.dnpm.gov.br/dnpm/paginas/balanco-mineral/arquivos/balanco-mineral-brasileiro2001-titanio>. Acesso em: 7 set. 2018.

MAZIERO, R.; BONONI, V. L.; CAPELARI, M. Cultivo e produtividade de Pleurotus ostreatus var. Florida em Mogi das Cruzes, Brasil. Hoehnea, São Paulo, v. 19, n. 1, p. 1-7, 1990.

MEIRELLES, G. Até 2021, indústria de celulose deverá investir R\$2,2 bi. Valor Econômico, São Paulo, jun. 2018. Disponível em: <http://www.sinpacel.org.br/informativos/2018/709/ate2021. pdf $>$. Acesso em: 8 set. 2018.

OEI, P. 0 cultivo de cogumelos em pequena escala: pleuroto, shiitake e orelha-de-pau. Wageningen: Fundação Agromisa e CTA, 2006. p. 18-36.

OLIVEIRA, P.; CALADO, M. L.; ROSADO, J. Etnomicologia: uma breve introdução. In: DIAS, A. S. (Ed.). Etnobotânica, Perspectivas, história e utilizações. Évora: Universidade de Évora, 2003. n. 4, p. 39-44.

PEDRA, W. N.; MARINO, R. H. Cultivo axênico de Pleurotus spp. em serragem da casca de coco (Cocos nucifera Linn.) suplementada com farelo de arroz e/ou de trigo. Arquivos do Instituto Biológico, São Paulo, v. 73, n. 2, p. 219-225, 2006. Disponível em: <http://www.biologico.sp.gov.br/uploads/ docs/arq/V73_2/pedra.PDF>. Acesso em: 7 set. 2018.

PEIXOTO, E. M. A. Silício. Química nova na escola, São Paulo, nov. 2001. Disponível em: < http:// qnesc.sbq.org.br/online/qnesc14/v14a12.pdf>. Acesso em: 20 set. 2014.

PICCININ, E. Cultura do cogumelo Shiitake (Lentinula edodes) em toras de eucalipto: teoria e prática. Piracicaba: ESALQ, 2000. p. 31-35.

PINTO, T. P. Gestão ambiental de resíduos da construção civil: a experiência do Sinduscon-SP. São Paulo: Obra Limpa: I\&T: Sinduscon-SP, 2005. p. 14-21.

RAGUNATHAN, R.; GURUSAMY, R.; ALANISWAMY, M.; SWAMINATHAN, K. Cultivation of Pleurotus spp. on various agro-residues. Food Chemistry, Reading, v. 55, n. 1, p. 139-144, 1996. Disponível em: <https://www.sciencedirect.com/science/article/pii/0308814695000798>. Acesso em: 7 set. 2018.

RAI, R. D. Cultivation of Reishi Mushroom (Ganoderma lucidum). Solan: NRCM, 2010. Disponível em: <http://nrcmushroom.org/Folder_Ganoderma.pdf>. Acesso em: 7 set. 2018.

ROJAS, A. C. B. Diversidade do gênero Ganoderma Karst. (Ganodermataceae) e atividade enzimática de espécies que ocorrem em leguminosas no Brasil e na Colômbia. 2016. 145 f. Tese (Doutorado em Biodiversidade Vegetal e Meio Ambiente) - Instituto de Botânica, Secretaria do Meio Ambiente, São Paulo, 2016. p. 11-18.

SALES-CAMPOS, C.; ANDRADE, M. C. N. Aproveitamento de resíduos madeireiros para o cultivo do cogumelo comestível Lentinus strigosus de ocorrência na Amazônia. Acta Amazonica, Petrópolis, v. 41, n. 1, p. 1-8, 2011. Disponível em: <http://www.scielo.br/scielo.php?pid=S0044$59672011000100001 \&$ script=sci_abstract\&tlng=pt $>$. Acesso em: 7 set. 2018. 
SANTOS, A. F. S.; DONADIA, J. T.; SANTOS, L. L. Ferro: Benefícios à saúde. In: MOSTRA ACADÊMICA UNIMEP, 8., 2010, Piracicaba. Anais... Pirabicaba: UNIMEP, 2010.

SANTOS, F. S.; SANTOS, A. L. A.; SANTOS, M. G. L. Análise ambiental do setor de papel e celulose no Brasil a partir da matriz SWOT. In: SEMANA DO ECONOMISTA, 3., 2013, Ilhéus. Anais... Ilhéus: UESC, 2013.

SILVA, R. R.; COELHO, G. D. Fungos: principais grupos e aplicações biotecnológicas. São Paulo: IBt, 2006. p. 18-19.

UNIVERSAL DE FERTILIZANTES S. A. Nutrientes: do que as plantas precisam?. Canoas: UNIFERTIL, 2012. Disponível em: <http://unifertil.com.br/wp-content/uploads/2018/04/Artigo-n\%C2\%BA-2Nutrientes-0-que-as-plantas-precisam.-min.pdf>. Acesso em: 7 set. 2018.

\section{Histórico editorial:}

Submetido em: 04/12/2016

Aceito em: 17/07/2017

Como citar:

ABNT

VALGINHAK, D. A.; SANTA, H. S. D. Potencial de utilização de resíduos da indústria papeleira como substratos para cultivo de Ganoderma lucidum (Reishi). Revista Agrogeoambiental, Pouso Alegre, v. 10, n. 3, p. 9-23, jul./set. DOI: http://dx.doi.org/10.18406/2316-1817v10n320181146

\section{APA}

VALGINHAK, D. A. \& SANTA, H. S. D. (2018). Potencial de utilização de resíduos da indústria papeleira como substratos para cultivo de Ganoderma lucidum (Reishi). Revista Agrogeoambiental, 10 (3), 9-23. DOI: http://dx.doi.org/10.18406/2316-1817v10n320181146

$\underline{\text { ISO }}$

VALGINHAK, D. A. e SANTA, H. S. D. Potencial de utilização de resíduos da indústria papeleira como substratos para cultivo de Ganoderma lucidum (Reishi). Revista Agrogeoambiental, 2018, vol. 10, n. 3, pp. 9-23. Eissn 2316-1817. DOI: http://dx.doi.org/10.18406/2316-1817v10n320181146

\section{VANCOUVER}

Valginhak DA, Santa HSD. Potencial de utilização de resíduos da indústria papeleira como substratos para cultivo de Ganoderma Iucidum (Reishi). Rev agrogeoambiental. 2018. jul./set.; 10(3): 9-23. DOI: http://dx.doi.org/10.18406/2316-1817v10n320181146 


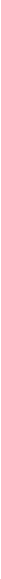

